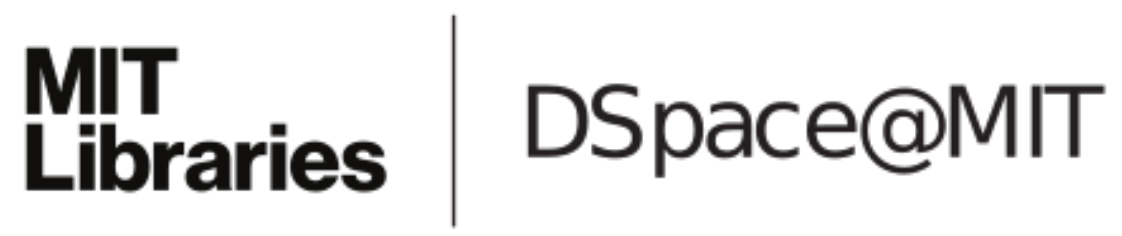

\author{
MIT Open Access Articles
}

Observations of Lower Hybrid Wave Absorption in the Scrape Off Layer of a Diverted Tokamak

The MIT Faculty has made this article openly available. Please share how this access benefits you. Your story matters.

Citation: Wallace, G. M. et al. "Observations of Lower Hybrid Wave Absorption in the Scrape Off Layer of a Diverted Tokamak." Gent (Belgium), 2009. 395-398. (2009 American Institute of Physics

As Published: http://dx.doi.org/10.1063/1.3273775

Publisher: American Institute of Physics

Persistent URL: http://hdl.handle.net/1721.1/67328

Version: Final published version: final published article, as it appeared in a journal, conference proceedings, or other formally published context

Terms of Use: Article is made available in accordance with the publisher's policy and may be subject to US copyright law. Please refer to the publisher's site for terms of use. 


\title{
Observations of Lower Hybrid Wave Absorption in the Scrape Off Layer of a Diverted Tokamak
}

\author{
G.M. Wallace*, R.R. Parker*, P.T. Bonoli*, R.W. Harvey ${ }^{\dagger}$, A.E. Schmidt*, \\ A.P. Smirnov ${ }^{\dagger}$, D.G. Whyte*, J.R. Wilson**, J.C. Wright* and S.J. Wukitch* \\ ${ }^{+}$MIT Plasma Science and Fusion Center, Cambridge, Massachusetts, USA \\ ${ }^{\dagger}$ CompX, Del Mar, California, USA \\ ${ }^{*}$ Princeton Plasma Physics Laboratory, Princeton, New Jersey, USA
}

\begin{abstract}
The Lower Hybrid Current Drive (LHCD) system on Alcator C-Mod is designed to drive off-axis current in the core plasma, e.g. $r / a \leq 0.8$, however several interesting observations indicate that the LH waves are depositing significant power in the Scrape Off Layer (SOL) in high density LMode discharges. These phenomena are observed in accessible plasmas below the so-called "density limit" attributed to Parametric Decay Instabilities as $\omega \rightarrow 2 \omega_{l h}$. Parallel current densities of $\sim 500$ $\mathrm{kA} / \mathrm{m}^{2}$ are measured on divertor Langmuir probes located just outside the separatrix during high power LH operation above $\bar{n}_{e} \sim 1 \times 10^{20} \mathrm{~m}^{-3}$. These SOL currents rise at the same density at which the line integrated hard X-ray Bremsstrahlung emission drops sharply, Ray tracing/Fokker-Planck simulations over-estimate the line integrated X-ray emissivity by a factor of $\sim 500$ at $\bar{n}_{e}=1.4 \times 10^{20}$ $\mathrm{m}^{-3}$ as compared to a factor of $3-4$ at $\bar{n}_{e}=0.6 \times 10^{20} \mathrm{~m}^{-3}$. Ray tracing simulations including a realistic SOL show similar trends in X-ray emission compared with the experimental observations.
\end{abstract}

Keywords: Lower Hybrid Current Drive, Density Limit, Scrape Off Layer, Bremsstrahlung PACS: $52.35 . \mathrm{Hr}, 52.35 . \mathrm{Mw}, 52.55 . \mathrm{Wq}, 52.55 . \mathrm{Fa}, 52.70 . \mathrm{La}$

The Lower Hybrid Current Drive (LHCD) system on the Alcator C-Mod tokamak is designed to drive off axis current on closed flux surfaces to achieve non-inductive discharges in excess of 1.0 MA of plasma current. Since the current drive efficiency is proportional to $1 / n_{e}[1]$, low density discharges are clearly of interest, however the application of LHCD to discharges with improved performance (high energy confinement, bootstrap current, etc) requires a shift to higher density. Two fundamental limits to the application of LHCD at high density, the accessibility criterion limit and the so-called "density limit" attributed to Parametric Decay Instabilities (PDI), must be avoided in order to ensure effective current drive. Recent results from Alcator C-Mod described in this paper show that other effects can contribute to a significant reduction in the fast electron population at densities below the limits imposed by accessibility and PDI.

The Hard X-Ray (HXR) diagnostic on C-Mod [2] measures bremsstrahlung emission from non-thermal electrons generated by the LHCD system. HXR is used both for localizing the bremsstrahlung emission and for comparing the relative magnitude of the count rate between discharges. The count rate can be taken as a proxy for the population of the fast electron tail generated by LHCD and therefore also for current drive. A rough estimate predicts that the fast electron bremsstrahlung emission should be inversely proportional to plasma density. Figure 1 shows that the line integrated HXR count rates on the central chords fall at a rate much steeper than $1 / n_{e}$. This is particularly true for the $5.4 \mathrm{~T}, 800 \mathrm{kA}$ case, which deviates from the $1 / n_{e}$ curve starting near $\bar{n}_{e} \sim 7 \times 10^{19}$ 

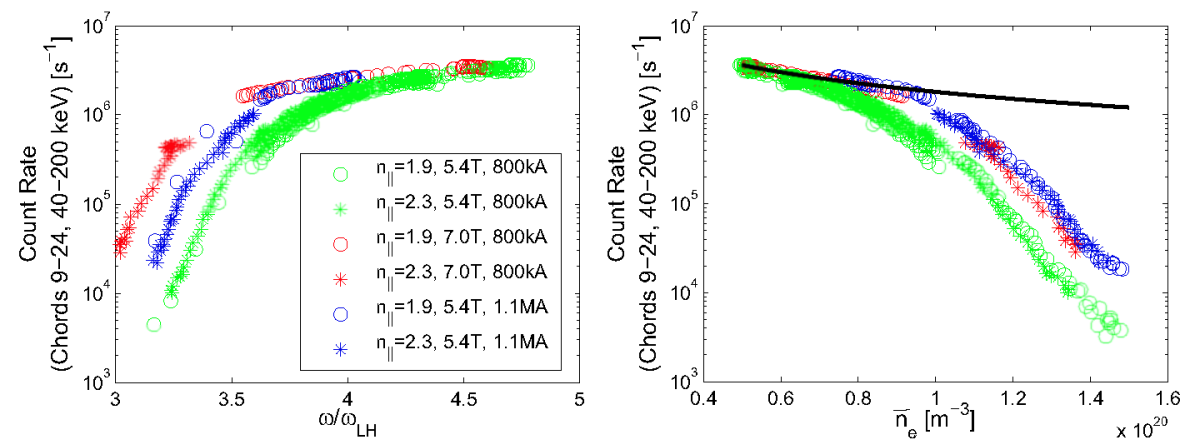

FIGURE 1. Line averaged density and $\omega / \omega_{L H}$ versus line integrated $\mathrm{X}$-ray emission on the central chords. Data is plotted for $\mathrm{X}$-rays between 40 and $200 \mathrm{keV}$. The black curve represents a $1 / n_{e}$ falloff. The count rates in this figure are normalized to $\sqrt{P_{L H}}$.

$\mathrm{m}^{-3}$. The data at higher magnetic field and plasma current drop more slowly as density rises, but are still two orders of magnitude lower than the $1 / n_{e}$ prediction at $1.5 \times 10^{20}$ $\mathrm{m}^{-3}$.

Although a violation of the accessibility condition would appear to explain the results of Figure 1, there are several points which call this explanation into question. First, the data taken at $n_{\|}=2.3$ and 1.9 do not show a measurable difference in count rate at any density. In addition, the accessibility criterion is satisfied everywhere in the plasma for a launched $n_{\|}$of 1.94 and 2.33, even at $\bar{n}_{e}=1.5 \times 10^{20} \mathrm{~m}^{-3}$ and $B_{\phi}=5.4 \mathrm{~T}$. Previous experiments have shown no accessibility problems for $n_{\mid \text {launch }} \geq n_{\mid \text {crit }}-0.05$ [3].

The "density limit" observed on previous experiments $[4,5]$ has been attributed to Parametric Decay Instabilities (PDI) as $\omega \rightarrow 2 \omega_{L H}$. The bremsstrahlung data from CMod, however shows a precipitous drop in emission below $\omega / \omega_{L H} \sim 3.75$, as can be seen in Figure 1. All of the data from C-Mod occurs at values of $\omega / \omega_{L H}$ in excess of 3, for which there should be no issue with PDI based on earlier results. The discrepancies between the data at 5.4T and 7.0 T in the left panel of Figure 1 show that bremsstrahlung emission is not a function of the lower hybrid frequency. The PDI level was recorded for many of the discharges shown by measuring the microwave frequency spectrum picked up by the LH Langmuir probes. Even at the highest densities (and consequently lowest values of $\left.\omega / \omega_{L H}\right)$ the strongest sideband is below $-20 \mathrm{~dB}$ relative to the pump.

For detecting the presence of LH waves in the SOL, one electrode from a reciprocating Gundestrup probe is connected through a $4.6 \mathrm{GHz}$ bandpass filter to a rectifier diode, the output of which is subsequently digitized. The reciprocating probe is located above the midplane in a location which is not magnetically connected to the LH antenna. Figure 2 shows a single in/out sweep of the reciprocating probe. Since the resonance cones leaving the LH antenna roughly follow the field lines, with $v_{g \perp} \ll v_{g \|}$, the reciprocating probe should not intersect waves leaving the antenna on the first pass to the separatrix. This suggests that the wave fields observed in the SOL may be due to waves trapped in the periphery of the plasma.

Flush-mounted Langmuir probes mounted in the upper and lower divertors can be 

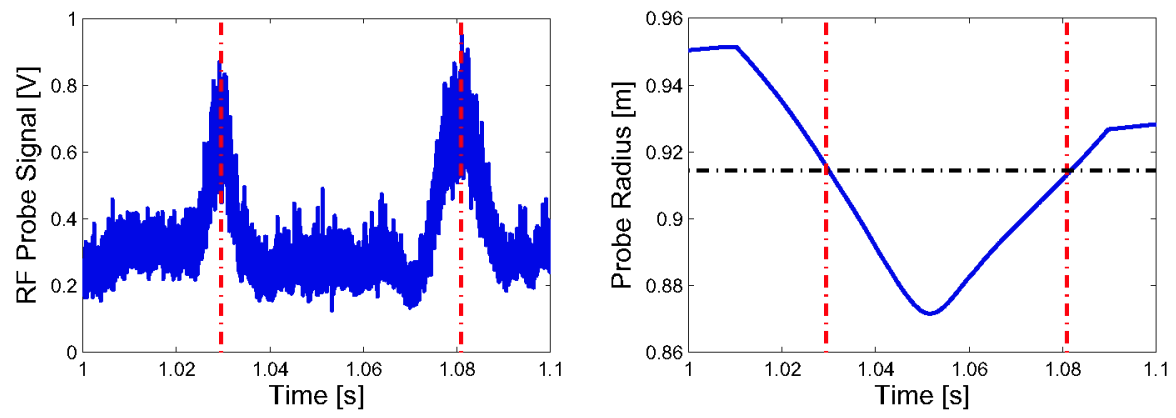

FIGURE 2. $4.6 \mathrm{GHz}$ wave fields in the SOL picked up by the A-port reciprocating Langmuir probe. One electrode of the probe is connected through a $4.6 \mathrm{GHz}$ bandpass filter to a RF diode detector.
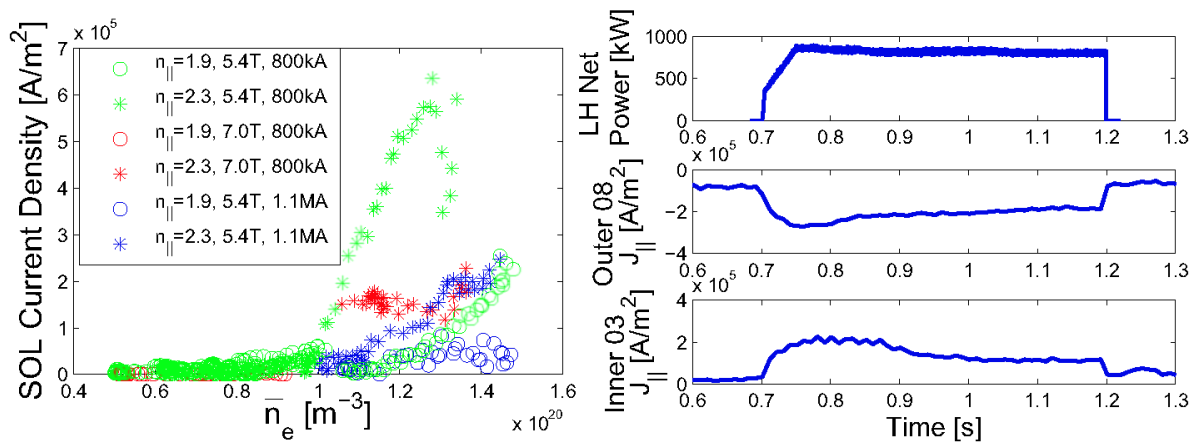

FIGURE 3. SOL current density as a function of $\bar{n}_{e}$. (Left) SOL currents observed during LHCD at high density in Lower Single Null. Equal and opposite $J_{\|}$are observed on the inner and outer divertor probes. The SOL current is flowing in the same toroidal direction as the main plasma current. (Right)

used to measure the parallel current density on open field lines. In normal operation, $J_{\|}$ measured by the divertor probes is less than $\sim 1 \times 10^{5} \mathrm{~A} / \mathrm{m}^{2}$ and is similar in magnitude on all of the probes. During high power LHCD operation at $\bar{n}_{e}>1 \times 10^{20} \mathrm{~m}^{-3}$, however, the magnitude of $J_{\|}$measured by some probes increases dramatically. Figure 3 shows the currents for probes on the inner and outer side of the lower divertor in a lower null discharge. The two probes, which lie on approximately the same flux surface, see currents which are nearly the same in magnitude and opposite in polarity. The polarity indicates that the current flowing between the two probes is in the direction of $I_{p}$, leaving the outer divertor, traveling up and around the top of the plasma, and returning to the vacuum vessel wall on the inner divertor.

The GENRAY/CQL3D ray tracing/Fokker-Planck solver simulation package $[6,7]$ predicts X-ray emissivity 2-3 orders of magnitude greater than experimental results at $1.5 \times 10^{20} \mathrm{~m}^{-3}$ when a SOL model is not included as shown in the left panel of Figure 4. These predictions are in qualitative agreement with the $1 / n_{e}$ estimate. The inclusion 

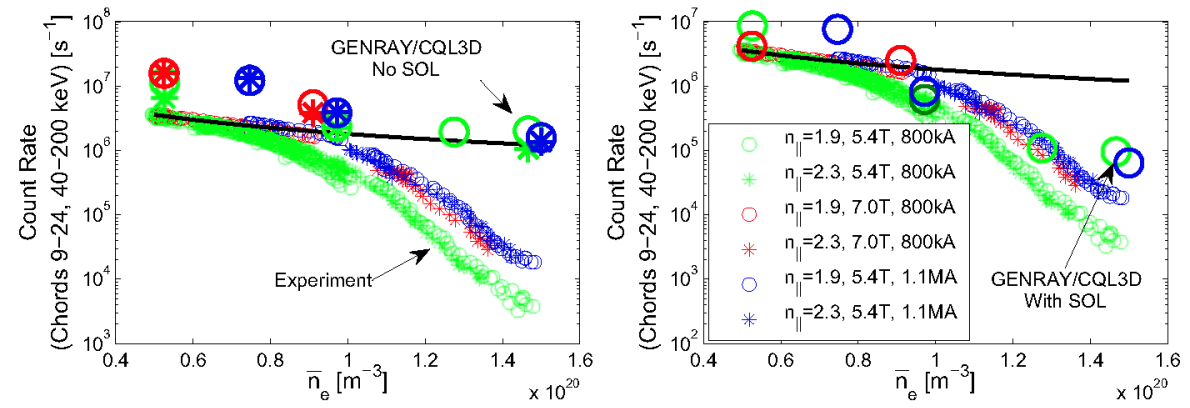

FIGURE 4. Simulated X-ray emission without a SOL included in the model and experimental data. (left) Simulated X-ray emission with a SOL included in the model and experimental data. (right)

of a SOL in the model allows for power to be absorbed through collisional damping outside the confined plasma and thus not contribute to the fast electron population in the plasma. Although CQL3D does not include open field lines in its calculation of quasi-linear damping, the collisional damping of LH waves in the SOL [8] is sufficient to significantly reduce the power absorbed inside the separatrix and thus the emissivity calculated by the CQL3D synthetic diagnostic. The right panel of Figure 4 shows the predicted X-ray emission with a temperature e-folding length of $0.005 \mathrm{~m}$ and a minimum temperature of $5 \mathrm{eV}$ in the $\mathrm{SOL}$, which agree with experimental values within the error of the measurement. This SOL model gives much better agreement across the entire density range for all values of magnetic field and plasma current. The increased emissivity at higher magnetic field and plasma current may be due to slightly higher temperatures at the separatrix which in turn reduces the collisional absorption in the SOL.

\section{ACKNOWLEDGMENTS}

Thanks to Dave Gwinn, Dave Johnson, Atma Kanokia, Pat MacGibbon, George MacKay, and Dave Terry for keeping the LHCD system running. This work supported by USDOE contracts DE-AC02-76CH03073 and DE-FC02-99ER54512.

\section{REFERENCES}

1. N. J. Fisch, Reviews of Modern Physics 59, 175-234 (1987).

2. J. Liptac, R. Parker, V. Tang, Y. Peysson, and J. Decker, Review of Scientific Instruments 77, 103504 (2006).

3. V. Pericoli-Ridolfini, et al., Phys. Rev. Lett. 82, 93-96 (1999).

4. Y. Takase, M. Porkolab, J. J. Schuss, R. L. Watterson, et al., Physics of Fluids 28, 983-994 (1985).

5. W. Hooke, Plasma Physics and Controlled Fusion 26, 133-144 (1984).

6. A. P. Smirnov, and R. Harvey, Bulletin of the American Physical Society (1995).

7. R. W. Harvey, and M. McCoy, Proceedings of the IAEA Technical Committee Meeting on Simulation and Modeling of Thermonuclear Plasmas (1992).

8. P. T. Bonoli, and R. C. Englade, Physics of Fluids 29, 2937-2950 (1986). 